\section{Histoire Épistémologie Langage}

42-1 | 2020

La grammaire arabe étendue

\title{
Hirschkop, Ken. 2019. Linguistic turns. 1890-1950
}

Oxford : Oxford University Press. xiv-323 p.

\section{Nick Riemer}

\section{(2) OpenEdition}

\section{Journals}

Édition électronique

URL : https://journals.openedition.org/hel/627

DOI : $10.4000 /$ hel.627

ISSN : 1638-1580

Éditeur

Société d'histoire et d'épistémologie des sciences du langage

Édition imprimée

Date de publication : 28 septembre 2020

Pagination : 186-190

ISSN : 0750-8069

Référence électronique

Nick Riemer, "Hirschkop, Ken. 2019. Linguistic turns. 1890-1950», Histoire Épistémologie Langage [En ligne], 42-1 | 2020, mis en ligne le 28 octobre 2021, consulté le 29 octobre 2021. URL : http:// journals.openedition.org/hel/627 ; DOI : https://doi.org/10.4000/hel.627

Ce document a été généré automatiquement le 29 octobre 2021

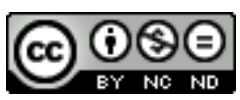

HEL is licensed under a Creative Commons Attribution-NonCommercial-NoDerivatives 4.0 International License 


\title{
Hirschkop, Ken. 2019. Linguistic turns. 1890-1950
}

Oxford : Oxford University Press. xiv-323 p.

\author{
Nick Riemer
}

\section{RÉFÉRENCE}

Hirschkop, Ken. 2019. Linguistic turns. 1890-1950. Oxford : Oxford University Press. xiv-323 p. ISBN 978-0-19-874577-8.

1 Ken Hirschkop (H.) propose dans Linguistic turns (LT) une analyse riche, stimulante et novatrice de travaux théoriques de penseurs importants, surtout anglais, allemands, français et russes, issus de champs disciplinaires différents et portant sur des questions de langue et de langage entre la dernière décennie du XIX ${ }^{e}$ siècle et le milieu du siècle suivant.

Dans une période caractérisée par les débuts d'une réflexion explicite sur les rapports entre le langage et la société, $\mathrm{H}$. fait une lecture socio-politique très subtile et révélatrice de textes pour la plupart classiques en philosophie, études littéraires et linguistique, entre le Saussure des années 1890 et les travaux d'Orwell, Austin et Wittgenstein dans les années 1940-1950. Ces textes n'abordent pas toujours les questions politiques de manière avouée. Mais, au-delà des grandes différences de cadre intellectuel entre, par exemple, Bakhtine, Benjamin, ou Ogden, H. décèle néanmoins la présence d'enjeux sociétaux déterminants pour les propos théoriques que tiennent les auteurs sur les structures et les pratiques langagières. Rejetant l'idée d'un seul "tournant linguistique " - terme rendu courant en philosophie par l'anthologie de Richard Rorty (1967) -, H. suit avec une grande érudition les évolutions de divers savants vers une conception qu'il dénomme «langage en tant que tel " (language as such), conception qui permet d'aborder, souvent sous forme tacite (on hésite à la qualifier d' " allégorique ", mais le terme paraît parfois juste), les problèmes d'un ordre social en pleine transformation, ou crise, démocratique. 
3 C'est chez Bakhtine, sujet d'un ouvrage précédent (Hirschkop 1999), que H. a fait pour la première fois l'hypothèse d'un « lien métonymique étroit entre le bon langage et la bonne politique»(p.185), constatant le passage effectué par le savant russe d'une philosophie éthique systématique dans les années 1920, conçue comme essentielle au « sauvetage » de l'Europe, vers une « identification du langage avec une conception de la vie sociale » (p. 138) dans les recherches sur Dostoïevski à la fin de la même décennie. Celui de Bakhtine n'était qu'un seul parmi toute une "constellation » de tournants linguistiques, aucunement limités à un seul champ disciplinaire et reflétant de manière très diverse les évolutions des sociétés modernes. Comme l'explique l'auteur (je traduis) :

le $\mathrm{XIX}^{\mathrm{e}}$ siècle avait effectué une identification entre les langues et les " peuples ». En même temps que ces " peuples » devenaient la substance des sociétés politiques et que la politique même devenait responsable de l'instauration et du maintien de l'ordre dans ces sociétés, les discussions sur la nature de l'ordre et du consentement linguistiques ont été investies d'une urgence toute particulière. (p. 153)

Ainsi, explique $\mathrm{H}$. de façon convaincante, au fil des décennies qu'il a étudiées, le langage servait dans la production théorique de métonymie pour des ensembles politiques et sociaux plus importants: à travers leurs propos sur le langage, les chercheurs traitaient en filigrane de la politique. Cette métonymie était à l'origine de divers surinvestissements théoriques. Certains, comme Benjamin, Bakhtine, Cassirer ou Šklovskij, ont trop misé sur le «langage en tant que tel», surestimant ainsi les possibilités politiques qu'il offrait, alors que d'autres, à l'instar de Saussure ou Ogden, se livraient à une vision plus pessimiste, exagérant combien les langues réelles sont défectueuses ou décevantes, trop rétives au changement. La lecture de Saussure et de Wittgenstein qu'avance $H$. est particulièrement éclairante :

5 Ces versions du « langage en tant que tel » n'étaient-elles pas un modèle, ou peut-être plus précisément, une vision de communauté et d'ordre social à un moment où l'ordre social semblait dépourvu de toute logique et de tout fondement? Ne fournissaient-elles pas un modèle qui acceptait l'individualisme atomistique de la société européenne, tout en démontrant comment celle-ci pourrait néanmoins être liée ensemble par un système ? Ce système, n'était-il pas une synthèse merveilleuse de la démocratie et de la quiétude politique ? Car « le langage » chez Saussure et Wittgenstein était, d'un côté, démocratique et auto-inventé - tout le monde participait à sa construction, et rien audelà ne le fondait - et, de l'autre côté, immunisé contre le débat public, dans la mesure où on ne pouvait pas proposer de raisons pour le changer. Dans une époque de crise, c'était une image d'équilibre social. (p. 103)

6 Au détour des huit chapitres, H. retrace la façon dont le langage permettait aux penseurs de la première moitié du siècle dernier de penser la politique, la nation, le consentement démocratique ou les fondements d'un ordre social, et d'analyser la bonne manière de prendre des décisions collectives. Pour certains d'entre eux, comme Saussure ou Wittgenstein, le langage fournissait un modèle de consensus et d'ordre. Pour d'autres - Ogden, Orwell, Bakhtine, Frege - et, parfois, pour Saussure aussi, il détenait une force motrice passionnelle, envisagée soit comme une menace à la démocratie, soit comme sa composante nécessaire.

7 Chez des penseurs célèbres dans les sciences humaines, tels Jean-François Lyotard, Ernesto Laclau et Chantal Mouffe ou Judith Butler, comme l'affirme H. dans sa préface, les déferlantes structuralistes et post-structuralistes ont transformé le langage en 
véhicule de propositions historiques ou politiques substantives, « comme si les théories du langage (Saussure, Wittgenstein, théorie des actes de parole, Foucault) avaient des affiliations politiques ou sociales» (p. viii). La prise de conscience à l'origine de $L T$, selon l'auteur, était l'idée selon laquelle « on a pu tirer des tendances et des conclusions politiques des textes théoriques sur le langage parce que des idées politiques et sociales étaient déjà implantées dans ceux-ci » (ibid.).

C'est donc à la recherche de ce contenu politique souvent implicite que $\mathrm{H}$. propose les analyses de $L T$. Il le fait à travers des lectures croisées d'une grande lucidité, détaillées et documentées, toujours alimentées par une connaissance profonde de la littérature scientifique concernant les penseurs qu'il aborde. $\mathrm{H}$. évoque souvent les travaux précédents avec beaucoup de générosité : le lecteur a l'impression qu'il n'a ignoré aucune piste soulevée dans le cours de ses recherches: chaque personnage, chaque thème a été étudié à fond (il faut signaler à ce propos que l'ouvrage est assorti d'un excursus intéressant - mais trop court - sur Sorel et Gramsci, dont les idées linguistiques, au lieu de découler de leurs positions politiques, influaient sur celles-ci). Une séquence particulièrement frappante (p. 65-80) mène de Wittgenstein à Lukács (les objets, d'ailleurs, d'une comparaison inattendue et pertinente), en passant, entre autres, par Benveniste, Habermas, Pearson, Rorty, Leavis et Williams. Effectivement, peu nombreux seront les lecteurs à même d'évaluer la justesse des propos de $\mathrm{H}$. dans leur totalité, vu l'érudition, l'originalité et la profondeur de ses analyses. L'auteur de ce compte rendu ne déroge aucunement à ce constat : j'ai appris beaucoup en lisant $L T$ et compte y revenir souvent.

9 H. explique que l'interprétation des tournants linguistiques qu'il défend ne peut trouver sa cohésion dans un seul récit continu : il raconte une « constellation » d'idées politiques sous la forme de théories linguistiques, et non une transmission historique ; de ce fait, $L T$ est organisé de manière thématique et "épisodique ». Les choix de l'auteur, parfaitement compréhensibles et pour la grande majorité pleinement justifiés, reflètent forcément une certaine conception de l'histoire intellectuelle quant aux questions de langue. Cette conception intègre Benjamin, Jakubinskij, Vinokur et Cassirer (ce dernier fait l'objet d'une discussion éclairante), tout en passant à côté de Merleau-Ponty, de Boas (exclu, sans doute, en raison de son immigration aux ÉtatsUnis; les articles recueillis dans Race and Democratic Society [Boas 1945] auraient pourtant fourni un point de départ pour des comparaisons intéressantes), ainsi que des théoriciens de deuxième plan, comme Damourette et Pichon, ou Polivanov - le vicecommissaire pour les affaires étrangères en 1917-1918-, comme de la plupart des linguistes examinés dans les travaux de Christopher Hutton (1999) sur la linguistique et le Troisième Reich. C'est donc une interprétation particulière que propose $\mathrm{H}$. de l'histoire des réflexions sur le langage: si quelque chose frappe dans $L T$, c'est la préférence qu'affiche l'auteur pour la politique comme thème souvent larvé des travaux sur le langage. Ainsi, certains penseurs qui abordent de front l'efficacité politique de la langue ne sont que rapidement discutés (c'est le cas de Vološinov) et certains thèmes sont même absents, comme les thèses de Polivanov (2014 [1931]) sur la linguistique "marxiste", qui ne fait l'objet que d'une brève référence (p. 214). De manière cohérente avec ce choix, mais au demeurant moins justifiée, la conception et les détails de la politique sont peu définis : l'histoire politique n'est guère brossée, même à gros traits, et le lecteur trouvera très peu de références à des événements historiques ou politiques précis, choix dont découle une certaine abstraction dans les analyses. $\mathrm{H}$. évite soigneusement aussi toute thématisation idéologique. Sa sympathie pour une 
certaine critique idéologique paraît néanmoins claire et certaines de ses lectures peuvent être lues comme un prolongement des positions de Richard Bauman et Charles L. Briggs (2003) dans leur étude essentielle Voices of Modernity. Language Ideologies and the Politics of Inequality. C'est le cas quand, par exemple, H. présente les projets de réforme linguistique dans la philosophie analytique comme des tentatives d'accaparement du débat politique par des clercs, ou voit dans l'objectivité scientifique un substitut aristocratique d'une sphère publique démocratique. $L T$ propose surtout une histoire intellectuelle du rôle des enjeux politiques à l'intérieur des idées sur le langage, pas du tout une lecture "externe", historique ou sociologique, de celles-ci. Il faut constater aussi que le détail et la rigueur bibliographique qui marquent l'investigation des idées linguistiques caractérisent moins la manière assez générale dont $\mathrm{H}$. aborde les questions politiques. Dans un travail qui propose d'étudier les rapports entre les idées sur la langue et les enjeux socio-politiques, cette frilosité est à regretter et laisse parfois le lecteur sur sa faim.

Ces réserves, secondaires, ne devraient surtout pas minorer l'accomplissement impressionnant que représente $L T$. L'ouvrage de H., passionnant, de grande envergure et très original, est le produit d'une érudition plurilingue mise au service d'un acharnement savant peu habituel et exprimée avec une élégance et une clarté de style admirables, étant donné surtout la densité des analyses. Chose rare, les thèmes dégagés, qui vont bien au-delà de ceux que j'ai pu relever ici, permettent d'aborder sous un jour tout à fait nouveau le récit de la pensée moderne sur le langage. L'histoire de la linguistique telle qu'on la pratique actuellement n'est sans doute ni suffisamment politique, ni suffisamment ouverte aux enjeux idéologiques. Si le livre de H. ne propose de suivre ni l'une ni l'autre de ces pistes en détail, il fournit néanmoins des analyses intellectuelles précieuses, et une cartographie du territoire très éclairante, à ceux qui voudraient prendre la relève. On peut donc espérer que $L T$ donnera lieu à des travaux d'approfondissement - de la part tant de H. lui-même, que d'autres chercheurs, que l'on souhaite nombreux - que l'ouvrage sera susceptible d'inspirer.

\section{BIBLIOGRAPHIE}

Bauman, R. et Briggs, Ch. L. 2003. Voices of modernity. Language ideologies and the politics of inequality. Cambridge : Cambridge University Press.

Boas, Fr. 1945. Race and democratic society. New York : J.J. Augustin.

Hirschkop, K. 1999. Mikhail Bakhtin: An aesthetic for democracy. Oxford : Oxford University Press.

Hutton, Chr. M. 1999. Linguistics and the Third Reich. Mother-tongue fascism, race and the science of language. Londres : Routledge.

Polivanov, E. D. 2014. Pour une linguistique marxiste. Éd. par Elena Simonato. Trad. par Elena Simonato et Patrick Sériot. Limoges : Lambert-Lucas.

Rorty, R., dir. 1967. The linguistic turn. Recent essays in philosophical method. Chicago : University of Chicago Press. 


\section{AUTEURS}

\section{NICK RIEMER}

The University of Sydney, HTL 